\title{
Optical Transmission Losses in Materials due to Repeated Impacts of Liquid Droplets
}

\author{
George S. Springer* and Cheng I. Yang † \\ University of Michican, Ann Arbor, Mich.
}

\begin{abstract}
The degradation of the optical transmittance of transparent and translucent materials subjected to repeated impingements of liquid droplets was investigated. An analytical model of the problem was developed, based on the postulate that changes in transmittance are due to cracks growing in the material. A simple expression was derived for predicting transmission losses in the material. The result obtained was compared to available experimental data, and reasonable agreement was found between the present result and the data.
\end{abstract}

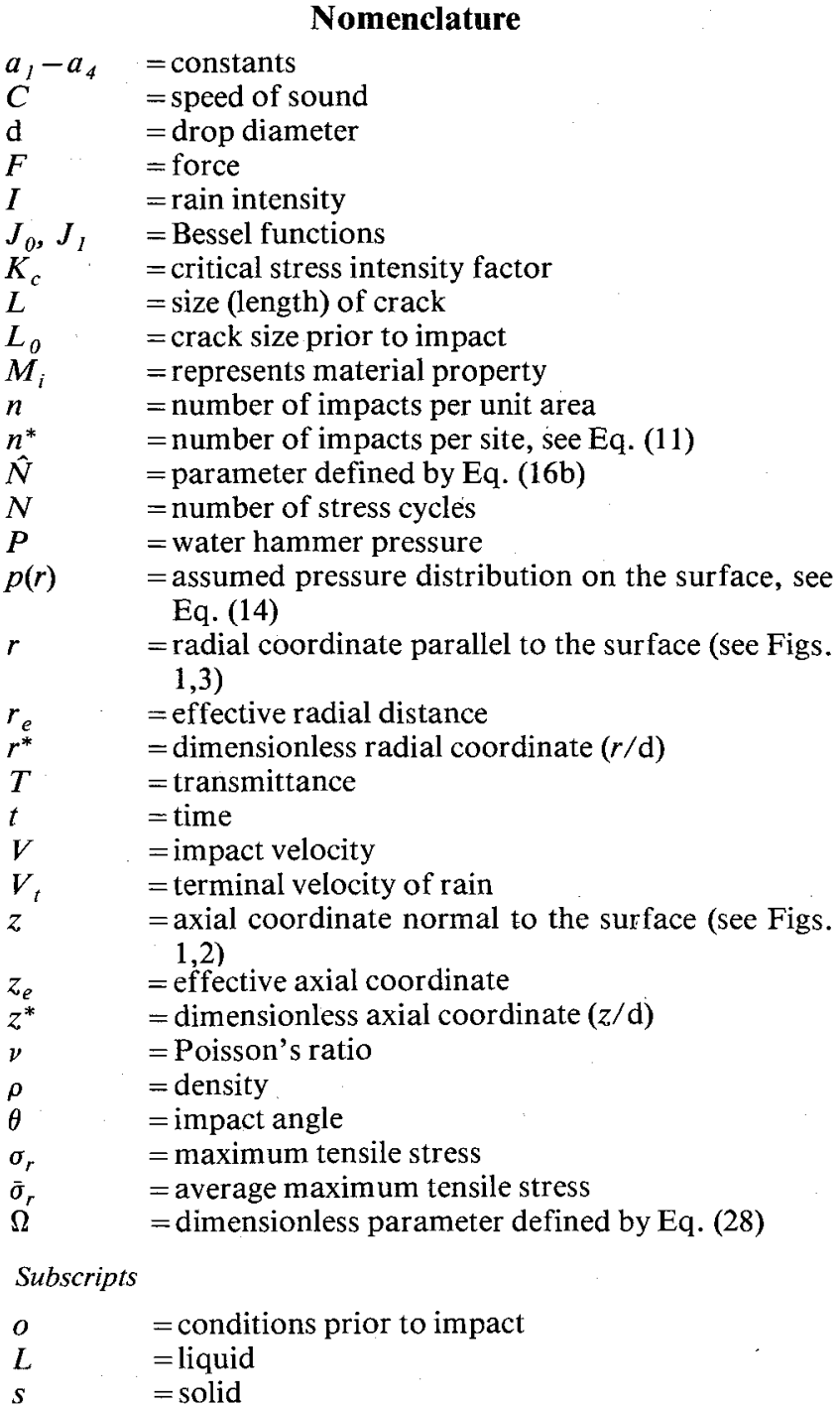

\section{Introduction}

IQUID droplets impinging upon a solid surface may cause significant damage and may render a component exposed to repeated impacts of droplets inefficient or

Received February 3, 1975; revision received March 31, 1975. The authors wish to thank Professor A. G. Atkins and G. F. Schmitt for their help.

Index categories: Aircraft Structural Materials; LV/M Simulation; Hypervelocity Impact.

*Professor, Department of Mechanical Engineering. Associate Fellow AIAA. even useless. For this reason, numerous investigations have been concerned with the effects of liquid impact on surfaces. However, most of the past studies focused on the mass loss of the material, because in many applications the damage is accompanied by a loss of mass (e.g., see the summaries in Refs. 1-3). Nevertheless, there are situations where a surface may become seriously damaged even before a mass loss is noticeable, as is the case, for example, of aircraft and spacecraft windows, whose transmission characteristics may change long before any measurable loss of mass occurs.

Although losses of optical transmittance are of great practical interest, to date only Hoff and Rieger ${ }^{4}$ and Schmitt ${ }^{5}$ have reported results pertinent to this problem. These investigators measured transmission losses in a few materials under selected conditions. An analytical model has not yet been devised which would describe the changes in the transmittance of transparent or translucent materials subjected to liquid impingement. The objective of this investigation was to develop such a model.

\section{The Problem}

The problem investigated is the following. Spherical liquid droplets of constant diameter $\mathrm{d}$ impinge repeatedly upon a semi-infinite, homogeneous material (Fig. 1). The angle of incidence of the droplets $\theta$, and the velocity of impact $V$ are taken to be constant. The spatial distribution of the droplets is considered uniform. The number of droplets impinging on unit area in time $t$ is denoted by $n$. For an idealized natural rain (composed of spherical droplets of uniform size) $n$ may be expressed as ${ }^{6}$

$$
n=(6 / \pi)\left[(V \cos \theta) I / V_{t} \mathrm{~d}^{3}\right] t
$$

where $I$ is the rain intensity and $V$, is the terminal velocity of the droplet. The impingement rate is assumed to be sufficiently low so that all the effects produced by the impact of one droplet diminish before the impact of the next droplet.

The pressure within the droplet varies both with position and with time. The pressure is taken to be constant at the liquid-solid interface, and is approximated by the water hammer pressure ${ }^{7}$

$$
P=\rho_{L} C_{L} V \cos \theta /\left(1+\rho_{L} C_{L} / \rho_{s} C_{s}\right) \quad r \leq \mathrm{d} / 2
$$

Fig. 1 Description of the problem.

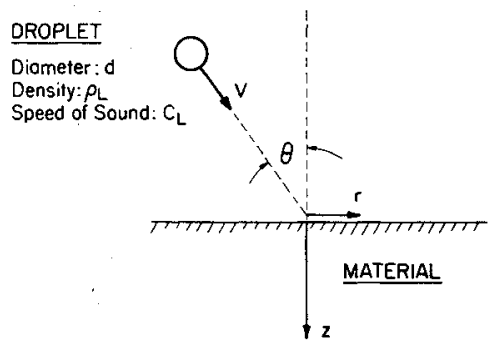




$$
P=0 \quad r>\mathrm{d} / 2
$$

$\rho$ is the density and $C$ the speed of sound. The subscripts $L$ and $s$ refer to the liquid and the solid, respectively. $r$ is a radial coordinate in the plane of the surface, with its origin at the center of impact.

The contact area between the droplet and the surface also varies with time. For simplicity we take this area to be constant, having the same value as the cross-sectional area of the droplet. Thus, the pressure acts within a radius of $r=\mathrm{d} / 2$. Outside of this radius the pressure is zero, see Eqs. (2a-2b). The force imparted to the surface by each droplet is

$$
F=P \pi \mathrm{d}^{2} / 4
$$

Although more accurate representations of pressure and force are possible, the accuracies afforded by the use of Eqs. (2) and (3) will suffice in the present analysis.

The forces created by the repeated droplet impacts damage the material. The damage may manifest itself in different forms. Here, we are concerned with optical transmission losses in translucent and transparent materials. The transmittance of the material depends on several parameters, including the absorptivity, reflectivity, the porosity of the material, and the sizes and orientations of the cracks on the surface and inside the material. The magnitudes of all of these parameters may change due to the liquid impact. It is likely, however, that change in crack size is the most important cause for change in transmittance. We assume, therefore, that changes in transmittance are caused only by changes in the sizes of the cracks. We further postulate that the transmittance $T$ varies inversely with the sizes of the cracks through which the light travels. Accordingly, the change in the transmittance is written as

or

$$
T / T_{0}=L_{0} / L
$$

$$
T / T_{0}=1 /\left[1+\left(L-L_{0}\right) / L_{0}\right]
$$

where $L$ is a length characteristic of the "sizes" of the cracks (Fig. 2). The subscript 0 refers to the conditions prior to impingement. In most practical situations cracks form only where crack nuclei are present, in which case $L_{0}$ corresponds to the sizes of the nuclei. Since the sizes of the nuclei are often unknown, the grain size (diameter) is frequently used for $L_{0}$. The problem at hand is to determine the change in the crack sizes $\left(L-L_{0}\right) / L_{0}$.
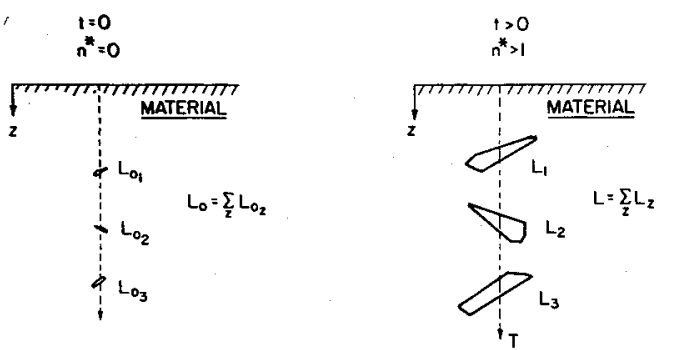

Fig. 2 The growth of cracks in the material because of the impingements of liquid droplets.

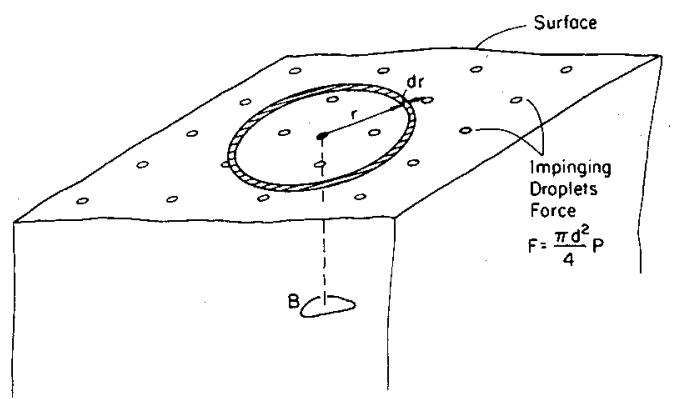

Fig. 3 Force distribution on the surface.

\section{Analysis}

Let us consider a single crack of size $L_{z}$, located at a distance $z$ below the surface, subjected to $N$ stress cycles (Fig. 3). The growth of this crack, caused by fatigue, depends on several parameters, of which the most significant are: a) the maximum stress occurring at the position of the crack $\sigma, b$ ) the instantaneous crack length $L_{z}$, and c) the appropriate material properties (denoted by $M_{1}, M_{2} \ldots$ ), i.e. ${ }^{8-11}$

$$
\mathrm{d} L_{z} / \mathrm{d} N=f\left(\sigma, L_{z}, M_{1}, M_{2} \ldots\right)
$$

The relative importance of the many different material properties on the growth of the crack has not yet been established. It is recognized, however, that the critical stress intensity factor $K_{c}$ plays an important role in the process of fatigue crack propagation. ${ }^{8,9,12,13}$ We adopt, therefore, $K_{c}$ as the representative material property. Equation (6) thus becomes

$$
\mathrm{d} L_{z} / \mathrm{d} N=f\left(\sigma, L_{z}, K_{c}\right)
$$

The form of the function $f$ in Eq. (7) is still subject to considerable debate, as manifested by the more than 30 "laws" proposed in recent years for relating $\mathrm{d} L / \mathrm{d} N$ to $\sigma, L_{z}$ and $K_{c} .{ }^{12}$ One of the most commonly accepted relationships for the fatigue crack growth rate is $8,10,12$

$$
\mathrm{d} L_{z} / \mathrm{d} N=a_{1}\left(\sigma \sqrt{L}_{z}\right)^{a_{2}}
$$

For lack of a generally accepted relationship we adopt the above expression, but in order to a) render the constant $a_{1}$ dimensionless and b) introduce $K_{c}$ (see Eq. 7), we rewrite Eq. (8) in the form

$$
\mathrm{d} L_{z} / L_{0} / \mathrm{d} N=a_{3}\left(\sigma \sqrt{L}_{z} / K_{c}\right)^{a_{2}}
$$

where $a_{3}$ and $a_{2}$ are as yet undetermined constants. For a given material $L_{0}$ and $K_{c}$ are constants and Eq. (9) reduces to Eq. (8). Equation (9) may be rearranged to yield

$$
\mathrm{d}\left(L_{z} / L_{0}\right) / \mathrm{d} N=a_{3}\left(\sqrt{L_{z} / L_{0}}\right)^{a} 2\left[\frac{\sigma \sqrt{L_{0}}}{K_{c}}\right)^{a_{2}}
$$

It is interesting to note that the dimensionless group $\sigma L_{0} / K_{\mathrm{c}}$ has been found to be of importance also in describing crack growth during machining and cutting. ${ }^{14,15}$

In the present problem the stresses in the material are caused by droplets impinging upon the surface. During one complete stress cycle one droplet impinges on each available "site," a "site" being defined as an area on the surface equal to the cross-sectional area of a droplet (Fig. 3). Since $n$ is the number of impacts per unit area, the number of impacts per site is

$$
n^{*}=\mathrm{n} \pi \mathrm{d}^{2} / 4
$$

Thus, in the present problem the number of stress cycles corresponds to the number of impacts per site $\left(N=n^{*}\right)$.

To obtain the total characteristic lengths of the cracks in the direction normal to the surface ( $L=\Sigma L_{z}$, Fig. 2) we would have to obtain an appropriate expression for $\sigma$ as a function of the position $r$ and $z$. This is a formidable, if not impossible, task because the stress depends in a complex manner on the sizes, shapes, orientations, and distribution of the cracks which are, of course, all unknown. To simplify the problem, we replace the individual cracks by a single equivalent crack which, for the purpose of evaluating the transmittance, has the same effective length as the sum of the lengths of the individual cracks. Thus, we write

$$
L=\Sigma L_{z}
$$


We assume, further, that this equivalent crack grows under the influence of an average tensile stress $\bar{\sigma}_{r}$. Accordingly, Eqs. (10) and (12) yield

$$
\mathrm{d}\left(L / L_{0}\right) \mathrm{d} n^{*}=a_{3}\left(\sqrt{L} / L_{0}\right)^{a_{2}}\left(\frac{\bar{\sigma}_{r} \sqrt{L_{0}}}{K_{c}}\right)^{a_{2}}
$$

Here $L_{0}$ is the size of the microstructural fault at which the equivalent crack is generated. The value of $L_{0}$ is taken to be equal to the grain diameter.

The calculations leading to the average stress $\bar{\sigma}_{r}$ are simplified considerably if, following the suggestion of Sneddon, ${ }^{16}$ the discontinuous boundary condition given by Eq. (2) is replaced by

$$
\left.p(r)=(P / 16) \mathrm{d}^{3} /\left[\left(\mathrm{d}^{2} / 4\right)+r^{2}\right)\right]^{3 / 2}
$$

Note that the total force on the surface due to $p(r)$ is the same as given by Eq. (3)

$$
\begin{aligned}
F=\int_{0}^{\infty} p(r) 2 \pi r \mathrm{~d} r & =\int_{0}^{\infty} \frac{P}{16} \frac{\mathrm{d}^{3}}{\left[\left(\mathrm{~d}^{2} / 4\right)+r^{2}\right]^{3 / 2}} \\
\times 2 \pi r \mathrm{~d} r & =P \frac{\pi \mathrm{d}^{2}}{4}
\end{aligned}
$$

The tensile stress in the material at a coordinate position $r$ and $z$ due to the above boundary condition, Eq. (14) is ${ }^{16}$

$$
\begin{aligned}
& \sigma_{r}(r, z)=\int_{0}^{\infty}(1-\gamma z) \hat{N} e^{-\gamma z} J_{o}(\gamma r) \mathrm{d} \gamma \\
& +\frac{1}{r} \int_{0}^{\infty} \hat{N}\left[z-(1-2 \nu) \frac{1}{\gamma}\right] e^{-\gamma z} J_{l}(\gamma r) \mathrm{d} \gamma
\end{aligned}
$$

where

$$
\hat{N} \equiv-P\left(\mathrm{~d}^{2} / 8\right) \gamma e^{-\gamma \mathrm{d} / 2}
$$

$J_{0}(\gamma r)$ and $J_{l}(\gamma r)$ are Bessel functions of the first kind, $\gamma$ is a dummy variable, and $\nu$ is Poisson ratio. Introducing the dimensionless coordinates

$$
r^{*} \equiv r / \mathrm{d} \quad z^{*} \equiv z / \mathrm{d}
$$

and integrating Eq. (16) we obtain

$$
\begin{aligned}
& \sigma_{r}\left(r^{*}, z^{*}\right)=-\frac{P}{2}\left[\frac{4 r^{* 2}\left(6 z^{*}+2\right)+2\left(1+2 z^{*}\right)}{\left[4 r^{* 2}+\left(1+2 z^{*}\right)^{2}\right]^{5 / 2}}\right. \\
& \left.-(1-2 v) \cdot\left[\frac{1}{4 r^{* 2}}-\frac{1+2 z^{*}}{4 r^{* 2}\left[1+2 z^{*}+4 r^{* 2}\right]^{3 / 2}}\right]\right]
\end{aligned}
$$

$\sigma_{r}$ is the tensile stress created by the impact of a single droplet impacting at a distance $r$ (or $r^{*}$ ) from the crack. Every droplet which falls at a radius $r$ produces the same stress at point $B$ (Fig. 3). The number of impacts on a $\mathrm{d} r$ wide annulus located at $r$ is

$$
n_{r}=(2 \pi r \mathrm{~d} r) n
$$

or, using Eq. (11)

$$
n_{r}=2 \pi r \mathrm{~d} r n^{*} / \pi \mathrm{d}^{2} / 4
$$

Thus, the stress due to the total number of droplets impacting the surface during one stress cycle (i.e., during one droplet on each site, $n^{*}=1$ ) is

$$
\sum_{\mathrm{n}} \sigma_{r}=\int_{0}^{r_{e}} \sigma_{r} \frac{2 \pi r}{\pi \mathrm{d}^{2} / 4} \mathrm{~d} r=8 \int_{0}^{r_{e}^{*}} \sigma_{r} r^{*} \mathrm{~d} r^{*}
$$

$r_{e}$ is defined as an effective distance from the impact point such that at $r_{e}$ the stress $\sigma_{r}$ is one per cent of that at $r=0$. Similarly, we define an effective length $z_{e}$, which is the distance below the surface where the stress is one per cent of that at the surface. The average stress is then expressed as

$$
\begin{aligned}
\bar{\sigma} & =\frac{1}{z_{e}} \int_{0}^{z_{e}} \int_{0}^{r_{e}} \sigma_{r} \frac{2 \pi r}{\pi \mathrm{d}^{2} / 4} \mathrm{~d} r \mathrm{~d} z \\
& =\frac{8}{z_{e}^{*}} \int_{0}^{z_{e}^{*}} \int_{0}^{r_{e}^{*}} \sigma_{r} r^{*} \mathrm{~d} r^{*} \mathrm{~d} r
\end{aligned}
$$

Substitution of Eq. (18) into Eq. (22) gives.

$$
\begin{gathered}
\bar{\sigma}_{r}=-\frac{P}{4 z_{0}^{*}} \int_{0}^{z_{e}^{*}} \int_{0}^{r_{e}^{*}}\left[\frac{4 r^{* 2}\left(6_{z}^{*}+2\right)+2\left(1+2 z^{*}\right)}{\left[4 r^{* 2}+\left(1+2 z^{*}\right)^{2}\right]^{5 / 2}}\right. \\
\left.-(1-2 \nu)\left[\frac{1}{4 r^{* 2}}-\frac{1+2 z^{*}}{4 r^{* 2}\left(1+2 z^{*}+4 r^{* 2}\right)^{3 / 2}}\right]\right] r^{*} \mathrm{~d} r^{*} \mathrm{~d} z^{*}
\end{gathered}
$$

The parameters $r_{e}^{*}$ and $z_{e}^{*}$ are of the same order of magnitude and are both of the order of 10. Integrating Eq. (23) and setting $r_{e}^{*}=z_{e}^{*} \ll 1$ we obtain

$$
\bar{\sigma}_{r}=P\left[1+(1-2 \nu)\left[2+\ln \frac{1+\sqrt{2}}{2}\right]\right]
$$

Equations (13) and (24) yield

$$
\begin{aligned}
& \frac{\mathrm{d}\left(L / L_{0}\right)}{\mathrm{d} n^{*}}=a_{3}\left(\sqrt { L / L _ { 0 } ) ^ { a _ { 2 } } } \left\{\frac{P \sqrt{L}}{K_{c}}\right.\right. \\
& \left.\left[1+(1-2 \nu)\left(2+\ln \frac{1+\sqrt{2}}{2}\right)\right]\right\}^{a_{2}}
\end{aligned}
$$

Integration of Eq. (25)

$$
\begin{aligned}
& \int_{I}^{L / L_{0}} \frac{\mathrm{d} L / L_{0}}{\left(L / L_{0}\right)^{a_{2} / 2}}=a_{3}\left[\frac{P \sqrt{L_{0}}}{K_{c}}\right. \\
& \left.\left[1+(1-2 \nu)\left(2+\ln \frac{1+\sqrt{2}}{2}\right)\right]\right]^{a_{2}} \int_{0}^{n^{*}} \mathrm{~d} n^{*}
\end{aligned}
$$

results in

$$
\frac{L-L_{0}}{L_{0}}=\left[1-a_{3}\left(\frac{a_{2}}{2}-1\right) \Omega^{a_{2}} n^{*}\right]^{1 /\left(1-a_{2} / 2\right)}-1
$$

$\Omega$ is a dimensionless parameter defined as

$$
\Omega \equiv \frac{P \sqrt{L}_{0}}{K_{c}}\left[1+(I-2 \nu)\left[2+\ln \frac{(1+\sqrt{2}}{2)}\right]\right]
$$

Equation (27) together with Eq. (5) gives the result sought

$$
T / T_{0}=\left(1-a_{4} \Omega^{a_{2}} n^{*}\right)^{2 /\left(a_{2}-2\right)}
$$

where $a_{4}$ is a constant replacing $a_{3}\left(a_{2} / 2-1\right)$.

\section{Discussion}

Equation (29) gives the loss of transmittance. The validity of this equation and the constants $a_{2}$ and $a_{4}$ must now be evaluated by comparing this result to experimental data. A comparison of Eq. (29) with the data of Hoff and Rieger ${ }^{4}$ and 
Table 1 Description of data and symbols used in Figs. 4 and 5, all tests were performed with water droplets

\begin{tabular}{|c|c|c|c|c|c|}
\hline $\begin{array}{l}\text { Material } \\
\text { reference }\end{array}$ & Symbol & $\begin{array}{l}\text { Impact velocity } \\
\qquad(\mathrm{m} / \mathrm{s})\end{array}$ & $\begin{array}{l}\text { Impact angle } \\
\text { (deg) }\end{array}$ & $\begin{array}{l}\text { Drop diam } \\
\quad(\mathrm{mm})\end{array}$ & $\begin{array}{l}\text { Rain intensity } \\
(\mathrm{mm} / \mathrm{hr})\end{array}$ \\
\hline $\begin{array}{l}\text { Polycarbonate } \\
\text { (Schmitt 1973) }\end{array}$ & i & $\begin{array}{l}270 \\
225 \\
180\end{array}$ & $\begin{array}{l}30,45,60,90 \\
30,45,60,90 \\
60,90\end{array}$ & 1.9 & 25 \\
\hline $\begin{array}{l}\text { Polysulfone } \\
\text { (Schmitt 1973) }\end{array}$ & $\begin{array}{l}\nabla \\
\square \\
\Delta\end{array}$ & $\begin{array}{l}270 \\
225 \\
180\end{array}$ & $\begin{array}{l}30,45,60,90 \\
30,45,60,90 \\
60,90\end{array}$ & 1.9 & 25 \\
\hline $\begin{array}{l}\text { Quartz } \\
\text { (Hoff \& Rieger 1972) }\end{array}$ & 0 & 450 & 90 & 1.2 & 180 \\
\hline $\begin{array}{l}\text { Magnesium oxide } \\
\text { (Hoff \& Rieger 1972) }\end{array}$ & $\otimes$ & 450 & 90 & 1.2 & 180 \\
\hline $\begin{array}{l}\text { Calcium aluminate } \\
\text { (Hoff \& Rieger 1972) }\end{array}$ & • & 450 & 90 & 1.2 & 180 \\
\hline
\end{tabular}

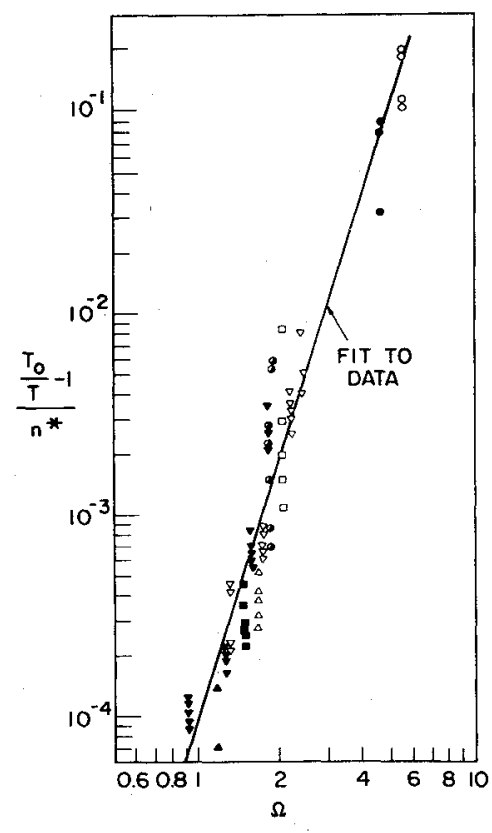

Fig. $4\left(T_{0} / T-1\right) / n^{*}$ vs $\Omega$. Symbols for data defined in Table 1.

Schmitt ${ }^{5}$ indicates that the transmission loss $T / T_{0}$ varies almost linearly with the number of impacts $n^{*}$. This suggests that the exponent $2 /\left(a_{2}-2\right)$ is unity and the constant is $a_{4}=4$. We also observe that at the early stages of the process $T / T_{0}$ is close to unity $\left(T / T_{0} \cong 1\right)$ and $a_{4} \Omega^{4} n^{*}$ is small compared to unity. Equation (29) may thus be written as

$$
T / T_{0}=I /\left(I+a_{4} \Omega^{4} n^{*}\right)
$$

Note that this expression provides the correct upper and lower limits for the transmission loss. Prior to the impact $n^{*}=0$ and Eq. (30) give the correct value of $T / T_{0}=1$; after a very large number of impacts $n^{*} \rightarrow \infty$ and Eq. (30) yield the required result of $T / T_{0}=0$.

To determine the constant $a_{4}$ and to assess the validity of Eq. (30), Eq. (30) is written in the form

$$
\left(T / T_{0}-1\right) / n^{*}=a_{4} \Omega^{4}
$$

This relationship will prove to be correct if, on a log-log scale, a plot of $\left(T_{0} / T-1\right) / n^{*}$ vs $\Omega$ results in a straight line of slope 4. The equation of this line would provide the constant $a_{3}$.

The $T_{0} / T, n^{*}$, and $\Omega$ values deduced from the available experimental data (all obtained with water droplets) are shown in Fig. 4. The symbols used in this figure and the corres-
Table 2 Material properties used in the calculations

\begin{tabular}{lcccc}
\hline \hline Material & $\begin{array}{c}\text { Density } \\
\left(\mathrm{mkg} / \mathrm{cm}^{3}\right)\end{array}$ & $\begin{array}{c}\text { Speed of } \\
\text { sound } \\
\left(\mathrm{m} / \mathrm{s}^{\prime}\right)\end{array}$ & $\begin{array}{c}\text { Poisson's } \\
\text { ratio }(\nu)\end{array}$ & $\begin{array}{c}\sqrt{L_{0} / K_{c}} \\
\left(\mathrm{~m}^{2} / \mathrm{N}\right)\end{array}$ \\
\hline Polycarbonate & 1.2 & 1425 & 0.25 & $40 \times 10^{-10}$ \\
Polysulfone & 1.24 & 1425 & 0.4 & $80 \times 10^{-10}$ \\
Quartz & 2.5 & 5300 & 0.2 & $45 \times 10^{-10}$ \\
Magnesium oxide & 3.57 & 9100 & 0.2 & $1 \times 10^{-5}$ \\
Calcium aluminate & 2.95 & 9350 & 0.29 & $3 \times 10^{-5}$ \\
Water & 1.0 & 1460 & & \\
\hline
\end{tabular}

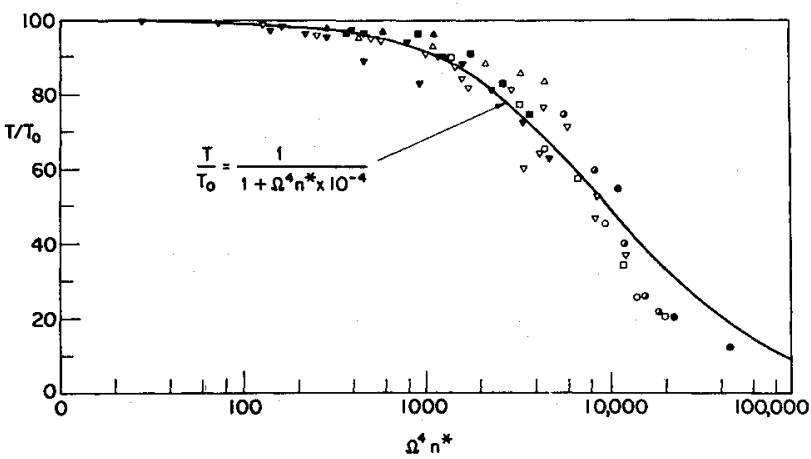

Fig. 5 Transmission loss $T / T_{0}$ vs $\Omega^{4} n^{*}$. Comparison of present model (solid line) with experimental data. Symbols for data defined in Table 1.

ponding experimental conditions are identified in Table 1. The material properties used in the calculations are listed in Table 2. It is seen from Fig. 4 that all the data can be correlated reasonably well by a straight line. The equation of this line, obtained by a least square fit of the data, gives the constant as $a_{4}=1 \times 10^{-4}$ and the exponent of $\Omega$ as 4 . The transmission loss (Eq. 29) is thus given by the expression

$$
T / T_{0}=1 /\left(1+\Omega^{4} n^{*} 10^{-4}\right)
$$

The final comparison between this equation and the data is shown in Fig. 5. The good correlation in Fig. 5 lends support to the validity of the model. It is conceivable, however, that the numerical values of $a_{2}$ and $a_{4}$ may need to be adjusted in the future as additional and more accurate data become available. There are, however, two points worthy of note. First, the change in transmittance varies with the fifth power of the impact velocity

$$
\left(T_{0} / T\right)-1 \sim \Omega^{4} n^{*} \sim P^{4} n^{*} \sim V^{4} n^{*} \sim V^{5}
$$


similarly to the mass loss rate of a material undergoing rain erosion, which also varies approximately with the fifth power of $V .6,17,18$ Second, $\left(T_{0} / T-1\right)$ varies with the fourth power of the stress; the fatigue crack growth rate is also nearly proportional to the fourth power of the stress over a wide range of conditions. 8,10

\section{References}

${ }^{1}$ Heymann, F.J., "A Survey of Clues to Relation between Erosion Rate and Impact Parameters," Proceedings of the Second Meersburg Conference on Rain Erosion and Allied Phenomena, Vol. 2, Aug. 1967, pp. 683-760.

${ }^{2}$ Springer, G.S. and Baxi, C.B., "A Model for Rain Erosion of Homogeneous Materials," Air Force Materials Lab., TR-72-106, June 1972, Wright-Patterson Air Force Base, Dayton, Ohio.

${ }^{3}$ Sims, M.G. and Trevett, P.W., Bibliography on Rain Erosion, Library Biby No. 327, May 1972, Royal Aircraft Establishment, Farnborough, England.

${ }^{4}$ Hoff, G. and Rieger, H., "Rain Erosion of Infrared Transmitting Materials," Proceedings of the Eleventh Symposium on Electromagnetic Windows, eds. N.E. Poulos and J.D. Walton, Vol. 1, Aug. 1972, pp. 93-97.

${ }^{5}$ Schmitt, Jr., G.F., "Effects of Rain Erosive Environment on the Transmission of Optically Transparent Plastic Materials," Air Force Materials Lab. TR-73-136, Aug. 1973, Wright-Patterson Air Force Base, Dayton, Ohio.

${ }^{6}$ Springer, G.S. and Baxi, C.B., "A Model for Rain Erosion of Homogeneous Materials," Erosion Wear and Interfaces with Corrosion, ASTM STP 567, Dec. 1974, pp. 106-127.

${ }^{7}$ Engel, O.G., "Waterdrop Collisions with Solid Surfaces," U.S. National Bureau of Standards, Journal of Research, Vol. 54, Jan. 1955, pp. 281-298.
${ }^{8}$ Knott, J.F., Fundamentals of Fracture Mechanics, Wiley, New York, 1973.

${ }^{9}$ McClintock, F.A., and Argon, A.S., Mechanical Behavior of Materials, Addison-Wesley, Reading Mass., 1966.

${ }^{10}$ Paris, P. and Erdogan, F., "A Critical Analysis of Crack Propagation Laws," ASME Transaction, Journal of Basic Engineering, Vol. 85, Dec. 1963, pp. 528-534.

${ }^{11}$ Wei, R.P., "Fracture Mechanics Approach to Fatigue Analysis in Design," Design Engineering Conference, Philadelphia, Pa., ASME Paper 73-DE-22, April 1973.

${ }^{12}$ Hoeppner, D.W. and Krupp, W.E., "Prediction of Component Life by Application of Fatigue Crack Growth Knowledge,' Fracture Mechanics and Its Application to Engineering Design, Union College, Schenectady, N.Y., 1974.

13 "Damage Tolerant Design Handbook" Air Force Materials Lab., MCIC-HB-01, Wright-Patterson Air Force Base, Dayton, Ohio, 1973.

${ }^{14}$ Atkins, A.G., "Fracture Toughness and Cutting," International Journal of Production Research, Vol. 12, Feb. 1974, pp. 263-274.

${ }^{15}$ Atkins, A.G., "A Dimensional Analysis for Machining to Include Fracture Toughness," Proceedings of the Second North American Metalworking Research Conference, Madison, Wis., Vol. 1, May 1974, pp. 398-407.

${ }^{16}$ Sneddon, I.N., Fourier Transforms, McGraw-Hill, New York, 1957.

${ }^{17}$ Springer, G.S., Yang, C.I. and Larsen, P.S., "Analysis of Rain Erosion of Coated Materials," Journal of Composite Materials, Vol. 8 , July 1974 , pp. 229-252.

${ }^{18}$ Springer, G.S. and Yang, C.I., "A Model for the Rain Erosion of Fiber Reinforced Composites," AlAA Journal, Vol. 13, July 1975, pp. 877-883.

\title{
From the AIAA Progress in Astronautics and Aeronautics Series...
}

\section{THERMAL CONTROL AND RADIATION—v. 31}

\author{
Edited by C.-L. Tien, University of California, Berkeley
}

Twenty-eight papers concern the most important advances in thermal control as related to spacecraft thermal design, and in radiation phenomena in the thermal environment of space, covering heat pipes, thermal control by other means, gaseous radiation, and surface radiation.

Heat pipe section examines characteristics of several wick materials, a self-priming pipe and development models, and the design and fabrication of a twelve-foot pipe for the Orbiting Astronomical Observatory C, and the 26-inch diode for the ATS-F Satellite.

Other thermal control methods examined include alloys, thermal control coatings, and plasma cleaning of such coatings. Papers examine the thermal contact resistance of bolted joints and electrical contracts, with role of surface roughness in thermal conductivity.

Gaseous radiation studies examine multidimensional heat transfer, thermal shielding by injection of absorbing gases into the boundary layer, and various gases as thermal absorbing media. Surface studies deal with real surface effects on roughened, real-time contaminated surfaces, and with new computational techniques to computer heat transfer for complex geometries, to enhance the capabilities and accuracy of radiation computing. 